\title{
Correction to: The Strengths and Difficulties Questionnaire (SDQ) in Africa: a scoping review of its application and validation
}

Nikhat Hoosen ${ }^{1,2}$, Eugene Lee Davids ${ }^{1 *}$ (D) Petrus J. de Vries ${ }^{1}$ and Maylene Shung-King ${ }^{1,2}$

\section{Correction to: Child Adolesc Psychiatry Ment Health (2018) 12:6 https://doi.org/10.1186/s13034-017-0212-1}

After publication of the article [1], it has been brought to our attention that the authors listed in Table 1 are in the wrong order. They should be listed as follows:

$$
\begin{aligned}
& \text { Kashala (2005) [25] } \\
& \text { Cluver (2006) [26] } \\
& \text { Kashala (2006) [27] } \\
& \text { Cluver (2007) [28] } \\
& \text { Menon (2007) [29] } \\
& \text { Okello (2007) [30] } \\
& \text { Cluver (2008) [31] } \\
& \text { Cluver (2009) [77] } \\
& \text { Doku (2009) [34] } \\
& \text { Elhamid (2009) [32] } \\
& \text { Menon (2009) [35] } \\
& \text { Bakare (2010) [36] } \\
& \text { Doku (2010) [37] } \\
& \text { Mueller (2011) [38] } \\
& \text { Puffer (2012) [39] } \\
& \text { Abbo (2013) [40] } \\
& \text { Atilola (2013) [41] } \\
& \text { Cortina (2013) [42] } \\
& \text { Kinyanda (2013) [43] } \\
& \text { Marais (2013) [44] } \\
& \text { Bhana (2014) [45] } \\
& \text { Devries (2014) [46] } \\
& \text { Escueta (2014) [47] } \\
& \text { Lachman (2014) [48] }
\end{aligned}
$$

\author{
Marais (2014) [49] \\ Skeen (2014) [51] \\ Sharp (2014) [50] \\ Waller (2014) [52] \\ Asante (2015) [53] \\ Casale (2015) [33] \\ Chirwa-Mwanza (2015) [62] \\ Collishaw (2015) [54] \\ Hermenau (2015) [55] \\ Mazzucato (2015) [56] \\ Okewole (2015) [78] \\ Pappin (2015) [57] \\ Profe (2015) [58] \\ Abdulmalik (2016) [59] \\ Bella-Awusah (2016) [60] \\ Bhana (2016) [61] \\ Cortina (2016) [63] \\ Dow (2016) [64] \\ Doku (2016) [65] \\ Hecker (2016) [66] \\ Hensels (2016) [67] \\ Lentoor (2016) [68] \\ Levetan (2016) [69] \\ Okewole (2016) [71] \\ Puffer (2016) [72] \\ Skeen (2016) [73] \\ Sherr (2016) [74] \\ Thumann (2016) [75] \\ Tucker (2016) [76] \\ Mazzucato (2017) [70]
}

\footnotetext{
*Correspondence: eugene.davids@uct.ac.za

${ }^{1}$ Adolescent Health Research Unit, Division of Child \& Adolescent

Psychiatry, University of Cape Town, 46 Sawkins Road, Rondebosch, Cape

Town 7700, South Africa

Full list of author information is available at the end of the article
} 
This has now been corrected in the original article.

\section{Author details}

${ }^{1}$ Adolescent Health Research Unit, Division of Child \& Adolescent Psychiatry, University of Cape Town, 46 Sawkins Road, Rondebosch, Cape Town 7700, South Africa. ${ }^{2}$ Health Policy and Systems Division, University of Cape Town, Cape Town, South Africa.

The original article can be found online at https://doi.org/10.1186/ s13034-017-0212-1.

\section{Publisher's Note}

Springer Nature remains neutral with regard to jurisdictional claims in published maps and institutional affiliations.
Received: 16 January 2018 Accepted: 17 January 2018

Published online: 31 January 2018

\section{Reference}

1. Hoosen N, Davids EL, de Vries PJ, Shung-King M. The Strengths and Difficulties Questionnaire (SDQ) in Africa: a scoping review of its application and validation. Child Adolesc Psychiatry Ment Health. 2018;12:6. https:// doi.org/10.1186/s13034-017-0212-1.

\section{Submit your next manuscript to BioMed Central} and we will help you at every step:

- We accept pre-submission inquiries

- Our selector tool helps you to find the most relevant journal

- We provide round the clock customer support

- Convenient online submission

- Thorough peer review

- Inclusion in PubMed and all major indexing services

- Maximum visibility for your research

Submit your manuscript at www.biomedcentral com/submit 